\title{
Imaging ventilation using I9F perfluorinated gas magnetic resonance imaging: strategies for imaging collateral ventilation
}

\begin{abstract}
Collateral Ventilation $(\mathrm{CV})$ has become an important clinical issue with the increasing use of bronchoscopic lung volume reduction (BLVR) using endobronchial valve surgery in patients with severe COPD. The endobronchial valve BLVR procedure often uses one way valves to occlude segmental bronchi in lung regions with severe overinflation resulting from airway narrowing and collapse during exhalation. For BLVR to succeed, CV to the treated region must be minimal or absent. Current approaches to evaluating $\mathrm{CV}$ for both planning and follow-up of BLVR procedures involve CT imaging to assess fissure closure. Current techniques to assess regional lung function (including CV) are limited. Standard pulmonary function testing involving analysis of inert gas wash-in/wash-out can only provide statistical distributions without anatomic correlates. Herein we propose the use of fluorine magnetic resonance imaging of biologically inert perfluorinated gas mixed with oxygen to evaluate regional ventilation, in particular, interlobar collateral ventilation. We have evaluated normal subjects and subjects diagnosed with chronic obstructive pulmonary disease and have observed gas transfer at lobar fissures consistent with collateral ventilation.
\end{abstract}

Keywords: ${ }^{19} \mathrm{~F}$ imaging, ventilation, collateral ventilation, physiology
Volume 8 Issue 2 - 202I

\author{
J Mammarappallil, ${ }^{3}$ NR Maclntyre, ${ }^{2}$ Kamran \\ Mahmood, ${ }^{2}$ S Womack, ${ }^{1,2} \mathrm{H}$ Cecil Charles ${ }^{1,3}$ \\ 'Radiology-Duke Image Analysis Laboratory, Duke University, \\ Durham, NC, United States \\ ${ }^{2}$ Div of Pulm \& Crit Care Med, Duke Univ Med Ctr, Durham, \\ NC, United States \\ ${ }^{3}$ Radiology, Duke University School of Medicine, Durham, NC, \\ United States
}

\begin{abstract}
Correspondence: $\mathrm{H}$ Cecil Charles, Duke Image Analysis Laboratory, Department of Radiology, Duke University School of Medicine, Suite 301, Hock Plaza, 2424 Erwin Road, Durham, NC, USA, 27705, Tel 919-684-792I, 919-260-560I, Fax 919-385975I,Email cecil.charles@duke.edu
\end{abstract}

Received: March 01, 202I | Published: April 01, 2021

\section{Abbreviations}

BLVR, bronchoscopic lung volume reduction; CAMRD, Center For Advanced Magnetic Resonance Development; COPD, chronic obstructive pulmonary disease; CT, computed tomography; CV, collateral ventilation; ${ }^{3} \mathrm{He}$, helium 3 isotope; IND, investigational new drug; IRB, institutional review board; MR(I), magnetic resonance (imaging); PFP, perfluoropropane, $\mathrm{C}_{3} \mathrm{~F}_{8}$ octafluoropropane; raw, airway resistance; SS, steady state images of ventilation imaging; $\mathrm{VH}$, ventilatory heterogeneity; WI, wash-in phase of ventilation imaging; $\mathrm{WO}$, wash-out phase of ventilation imaging; ${ }^{129} \mathrm{Xe}$, Xenon 129 isotope for hyperpolarized gas imaging; ${ }^{133} \mathrm{Xe}$, Xenon 133 radioisotope (used in Xenon Scintigraphy)

\section{Introduction - Collateral Ventilation}

Pulmonary ventilation is the process of moving gas between the environment and the pulmonary gas exchange regions (alveolarcapillary interface). It consists of convective or bulk flow gas movement through the 23 branches of the tracheo-bronchial tree and is coupled with diffusive gas mixing within millions of alveolar units. The distribution of ventilation among these units is determined by the gas driving pressures generated by respiratory muscles interacting with regional lung mechanics (i.e. resistances and compliances). Regional resistances and compliances are driven respectively by airway caliber (including mucus production and modulation by airway smooth muscles) and lung tissue elasticity. In normal lungs, the distribution of ventilation is "designed" to match the distribution of perfusion to assure optimal gas exchange at the alveolar-capillary interface.

Collateral ventilation (CV) occurs when gas exchange happens between one region of the lung to another region via several possible indirect pathways including interlobar fissure exchange, intrabronchiolar pathways of Martin, bronchiole-alveolar communications of Lambert and interalveolar pores of Kohn. ${ }^{1-6}$ Due to the increased airway resistance in these pathways, about $50 \mathrm{x}$ normal airway resistance (Raw), collateral ventilation is generally considered unimportant in normal lungs. However, there is good evidence that these indirect pathways may be more important in diseases such as emphysema where the direct pathways are damaged. ${ }^{4,6-8}$

$\mathrm{CV}$ has become an important clinical issue with the increasing use of bronchoscopic lung volume reduction (BLVR) using endobronchial valve surgery in patients with severe COPD. The endobronchial valve BLVR procedure often uses one way valves to occlude segmental bronchi in lung regions with severe overinflation resulting from airway narrowing and collapse during exhalation. For BLVR to succeed, CV to the treated region must be minimal or absent. Current approaches to evaluating CV for both planning and follow-up of BLVR procedures involve CT imaging to assess fissure closure. Pre-procedure this is generally followed by evaluation of regional inflation during bronchoscopy which can be cumbersome and time consuming. At the present time there is no optimal approach (imaging or otherwise) to guide which region(s) might be most suitable for the intervention. $^{9-14}$

\section{Approaches to assessing regional lung function (including CV)}

Current techniques to assess regional lung function (including $\mathrm{CV})$ are limited. Standard pulmonary function testing involving analysis of inert gas wash-in/wash-out can only provide statistical distributions without anatomic correlates. ${ }^{15,16}$ Planar nuclear Xenon scintigraphy yields gross ventilation distribution information, involves radioactive agents and provides only two-dimensional images. ${ }^{17,18}$ Chest computerized tomography can give high resolution, threedimensional images of anatomic structures only at end inspiration and end expiration, requires ionizing radiation and provides little functional information. ${ }^{19}$ 
Imaging the distribution of a gas throughout the pulmonary structures is often achieved using a single static breath-hold of the lung field that provides just a snapshot of the underlying physiological processes. ${ }^{20-26}$ However, pulmonary ventilation is a complex, dynamic process that controls and matches gas exchange with blood flow within regional lung units. Thus, detection of these localized changes that occur during ventilation in the normal lung and altered with the onset of early airway and parenchymal disease conditions are of increasing importance.

Magnetic resonance imaging (MRI) has great potential to address these shortfalls. MRI of lung airspaces, however, requires exogenous gaseous contrast agents much like other imaging strategies. These gases are needed to overcome the low MRI signal intensity, susceptibility and motion artifacts in aerated lungs that result from low proton density, numerous air-tissue interfaces and cardiac and respiratory motion. ${ }^{27}$ MRI with appropriate gaseous contrast agents thus can provide high resolution, three-dimensional quantitative images without the use of ionizing radiation. Collection of regional ventilation information using magnetic resonance imaging could prove beneficial and hence become heavily favored as an imaging modality for assessing the suitability and practicality of pharmaceutical and surgical interventions (including BLVR) designed to improve ventilation.

Detection of such abnormal ventilation such as $\mathrm{CV}$ with an imaging technology is challenged by issues of spatial and functional resolution. In the spatial domain computed tomography combined with various rendering strategies to evaluate interlobar fissure integrity is challenged by the issue of spatial rendering of two spatially intimate surfaces to find incomplete closure of such fissures. Unfortunately, functional evaluation using $\mathrm{CT}$ is challenged by the inability to measure ventilation, i.e. the air is not "colored" so temporal evaluation of the airways, i.e. ventilation) is not practical. 4DCT has been touted as a measure of lung function but is basically a measure of motion. Hyperpolarized gas has also been proposed but has been limited to evaluation of so called ventilation defects and/or apparent diffusion measurements. ${ }^{28,29}$

Evaluating intralobar and interlobar collateral ventilation is a challenge for any imaging modality. Such collateral ventilation only becomes important in regions of slow filling where Raw is substantially increased by disease processes. While the perfluorinated gas imaging shows regions likely due to interlobar $\mathrm{CV}$ and could be validated with either CT evaluation of fissure closure or invasive measures post endobronchial valve placement, this is more complicated for intralobar (intra-acinar) CV where the distinction between a very slow filling compartment and collateral ventilation is challenging as there is no alternative for validation compared to interlobar CV. It is likely that at distal regions of the airway tree the transition from slow filling compartments to gas exchange by intralobar $\mathrm{CV}$ represent a continuum of processes.

\section{Methods: |9-fluorine (I9F) enhanced MRI as a novel approach to evaluate regional ventilation}

Subjects in this report were selected from a larger study of subjects with normal lung function as well as subjects diagnosed with Chronic Obstructive Pulmonary Disease (COPD) funded in part by NIH/NHLBI R01-HL111294 (NCT 01640288). Subjects were consented per local and federal regulations and presented at the MR imaging suite (Center for Advanced MR Development, CAMRD) for the imaging procedure. To comply with local policies related to incorporating scheduling research studies in EPIC (Duke Maestro Care) a preliminary IRB approved limited verbal consent process was accomplished primarily to rule out any potential subjects with MRI contraindications. After verbal screening a preliminary visit for written consent was scheduled followed by screening per the protocol. Perfluoropropane (PFP- $\mathrm{C}_{3} \mathrm{~F}_{8}$ ) is a relatively inexpensive imaging technique enabling a multi-breath, dynamic steady state assessment of underlying gaseous distribution in the lung airspaces. Through exploitation of the large gyromagnetic ratio and relaxation properties of this gas, a temporal and spatial assessment of the underlying gaseous distribution is feasible. ${ }^{30,31}$ The applicability of the techniques has been previously demonstrated in animal models as well as in excised lungs. ${ }^{32,33}$ Recently, the first results in human subjects with normal and abnormal lung function using PFP gas in static single breath hold images have been presented, ${ }^{30}$ demonstrating the applicability of PFP enhanced MRI in assessing the gaseous distribution and ventilation defects in human subjects with varying degrees of lung function.

Fluorine MRI using exogenous gas mixtures offers significant advantages over current clinical lung function imaging with planar scintigraphy where the spatial/anatomic resolution precludes evaluation of collateral ventilation. In order to visualize and measure $\mathrm{CV}$ non-invasively, we need an imaging marker that can be visualized after switching from the imageable gas to breathing room air where slow filling/emptying compartments provide the residual imaging gas to other regions of the lung. Using perfluorinated gas imaging we can evaluate regional ventilation by collecting a series of short breath hold images while the subject breaths a biologically inert perfluorinated gas (perfluoropropane in this case) mixed with oxygen from which we demonstrate changes in reginal ventilation as shown in the montage in Figure 1

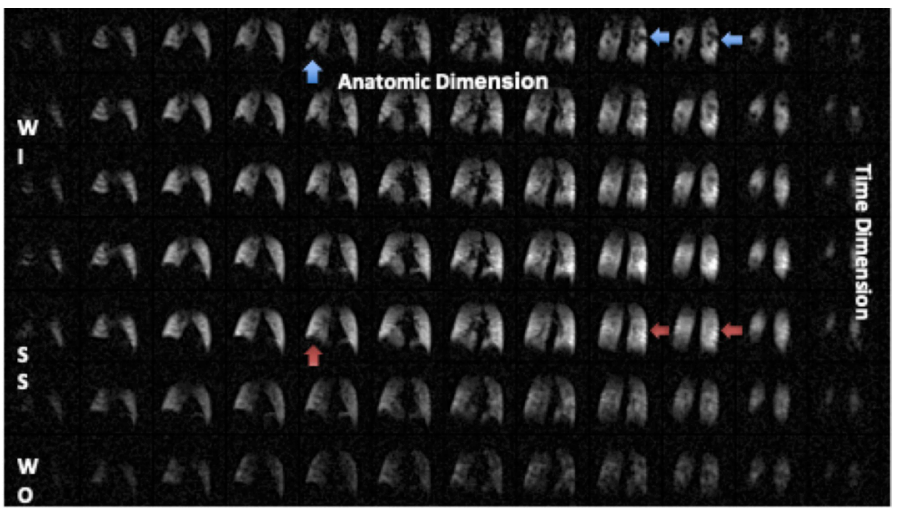

Figure I Montage of a Ventilation ${ }^{19} \mathrm{~F}$ MRI scan in a subject with COPD (Left to Right are 3D anatomic images, top to bottom represents 7 of 9 acquired time series volumes). In the first row (first breath-hold image, the blue arrows depict signal voids, often denoted as 'ventilation defects' by the hyperpolarized gas community. As is evident in this time series of images (top to bottom), these represent slow filling compartments (red arrows at steady state). The time series can be fit a model generating quantitative images of wash-in and washout time constants. NOTE:WI, wash-in; SS, steady state;WO, wash-out.

Using such a spatio-temporal set of images we can evaluate collateral ventilation by comparing the steady state image from the early washout image as shown in the schema in Figure 2. This is a unique feature of ${ }^{19} \mathrm{~F}$ gas MRI that is challenging with nuclear scintigraphy and difficult to impossible with hyperpolarized gases as the rare isotope gases such as ${ }^{3} \mathrm{He}$ and ${ }^{129} \mathrm{Xe}$ cannot be 'repolarized' in the lung airspaces. 


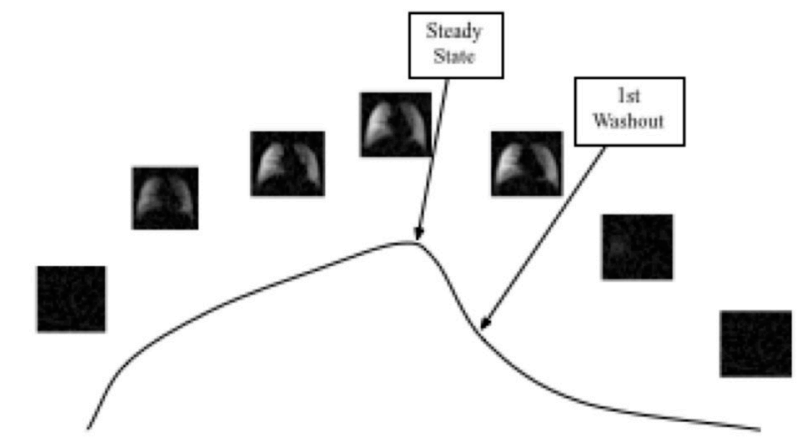

Wash-IN

Wash-OUT

Figure 2 Schema for wash-in, wash-out imaging and strategy for $\mathrm{CV}$ evaluation using the difference in the early washout and steady state images.
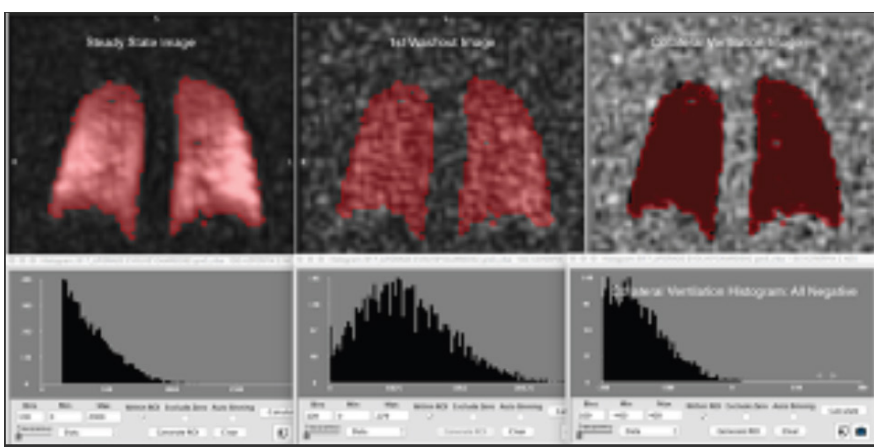

Figure 3 One slice from a 3D image set at steady state and early washout in a subject with normal lung function and the difference image showing only negative values (right) indicating absence of collateral ventilation.

We are able to quantitatively distinguish the range of slow to fast filling compartments using perfluorinated gas (perfluoropropane mixed with oxygen) imaging. We propose a strategy to evaluate and visualize collateral ventilation by subtracting the steady state image from the early washout image as shown in Figure 3 from a 43 year old normal subject (non-smoker). Figure 3 shows one slice from the subject where there are no positive values in the difference image. For example, the combination of the collateral ventilation map and the wash-in/wash-out maps and extracted information could provide a clearer framework for selection of the best lobe for BLVR. This is a unique feature of ${ }^{19} \mathrm{~F}$ gas MRI that is challenging with nuclear scintigraphy and difficult to impossible with hyperpolarized gases as the gases cannot be re-polarized in the lung airspaces.

Figure $4 \mathrm{a}$ and Figure $4 \mathrm{~b}$ show the same approach in a subject with COPD (GOLD 3 ) with a single slice (from the $3 \mathrm{D}$ volumetric set) with the images and histogram showing positive values in the difference image as well as a CT image at the same anatomic level supporting the strategy that there is gas exchange across the superior-middle lobe fissure in the right lung.

Figure 5 shows a montage of difference images from normal subjects with and without a history of smoking. In contrast Figure 6 shows a montage of difference images subjects with COPD. There are varying patterns of ventilation with clear indications of both interlobar and intralobar signal enhancement during wash-out on room air consistent with collateral ventilation.
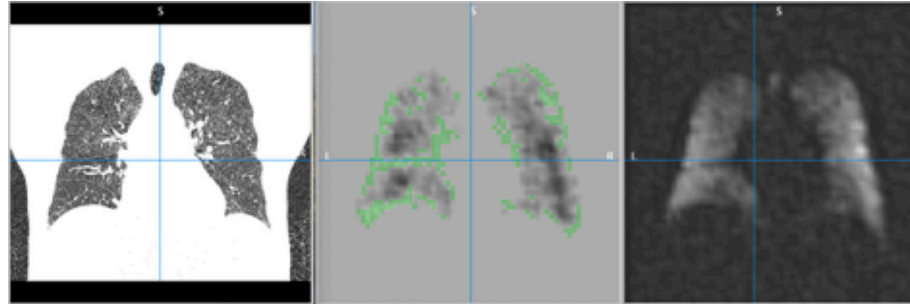

Figure 4a (left to right) CT (expire), difference image (EWO-SS) and SS gas image. The blue crosshairs are at the level of the superior to middle lobe fissure (right lung). The difference image shows positive values denoted by green overlay pixels described by the histogram of the slice in Figure $4 \mathrm{~b}$.

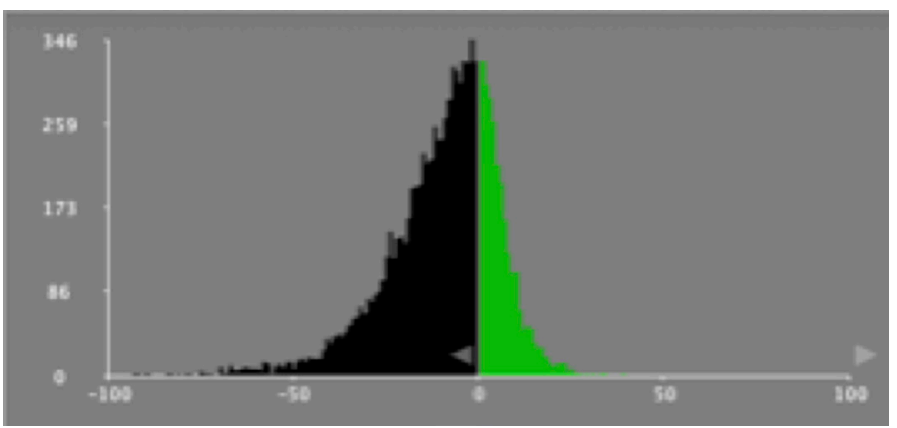

Figure 4b Histogram of the difference image in figure $4 a$ center depicting positive values not seen in normal lung difference images.
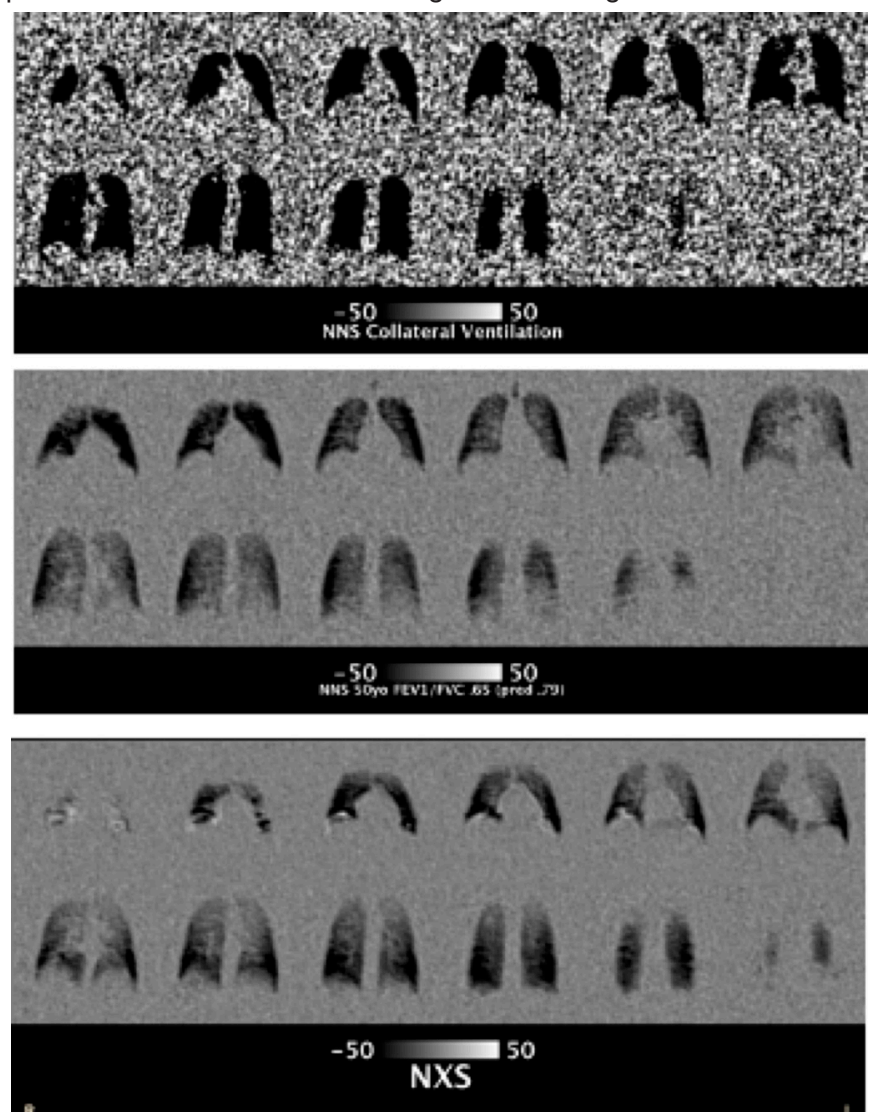

Figure 5 Examples of EWO - SS images in a Normal non-smoker (top), a normal non-smoker with $\mathrm{FEVI}=0.7$ (middle) and an ex-smoker with normal FEVI (>0.7) 

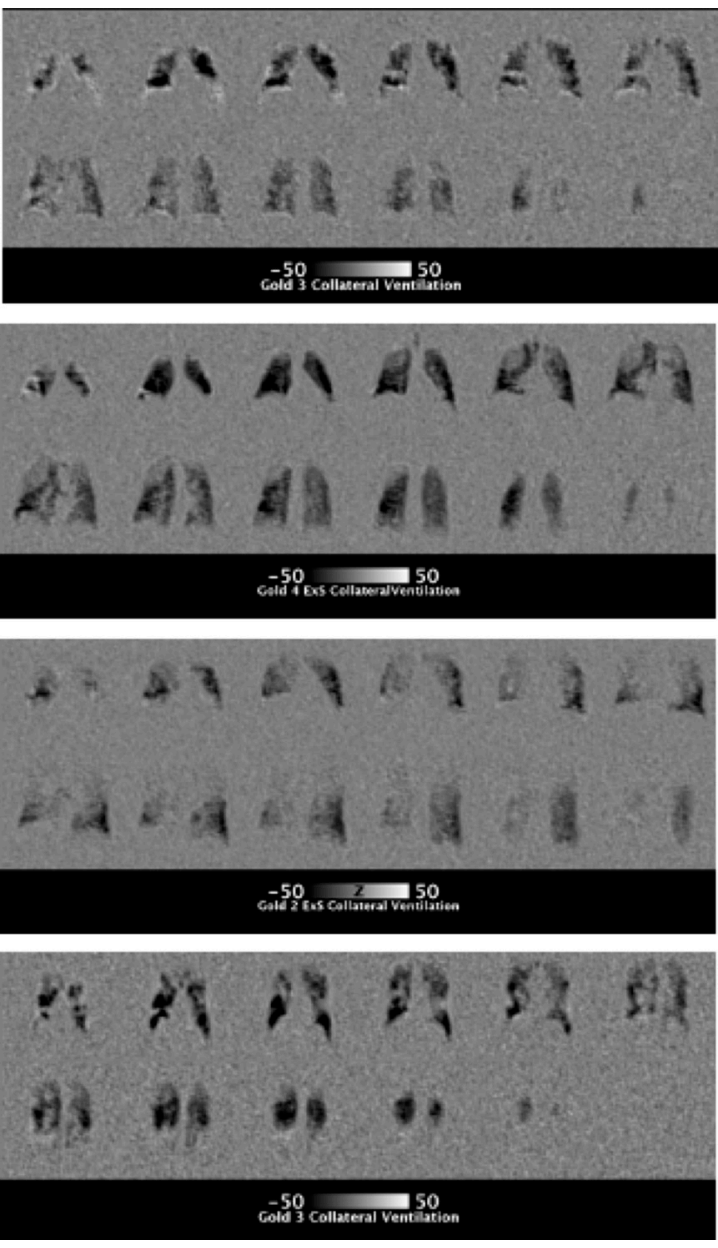

Figure 6 Subject with COPD (Gold 3) note interlobar signal in right lung (top), Subject with COPD (Gold 4) and large Bullae (upper middle), Subject with COPD (Gold 2, Ex-smoker) (lower middle) and Subject with COPD (Gold 3) (lower).

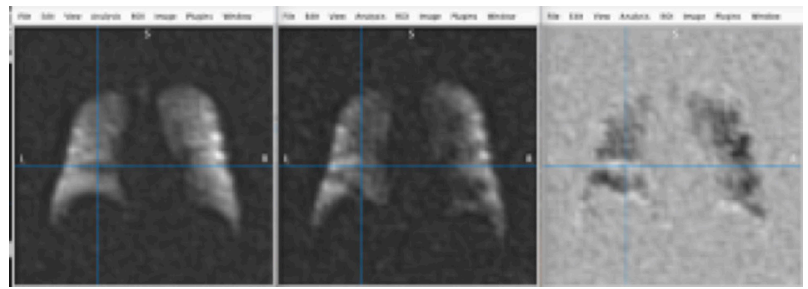

Figure 7a Steady state, Early Wash out and Difference image in subject with COPD.

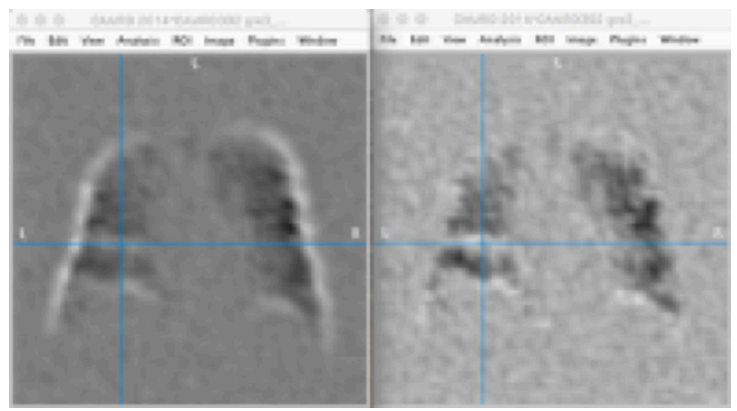

Figure 7b On the left the image shows a difference image where the inplane wash-out image has been scaled up by $10 \%$ (analogous to breath-hold differences) compared on the right to the difference image from Figure $7 \mathrm{a}$.

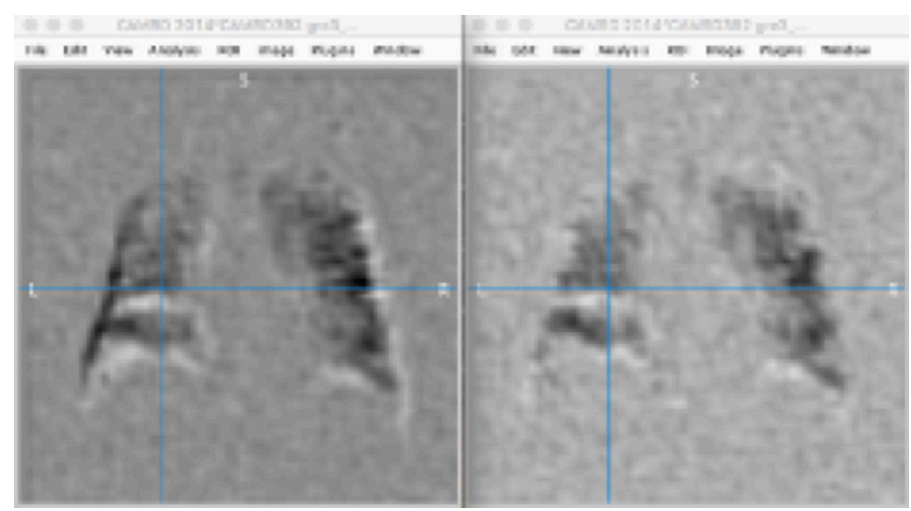

Figure 7c On the left the image shows a difference image where the in-plane wash-out image has been shifted by one pixel in the L-R direction and one pixel in the S-I direction compared on the right to the difference image from Figure 7a.

\section{Conclusion}

Some questions may arise about what we are 'seeing' in these images. Clearly in subjects with COPD there are slow filling compartments where the signal intensity continues to increase after the subject is switched to room air. This raises some issues, in particular, regarding intra-lobar collateral ventilation; are we just measuring very slow filling compartments although the subjects are breathing room air at the time of the washout images so any ${ }^{19} \mathrm{~F}$ gas present is also slowly emptying or coming from another region of the lung. A second possible issue is the possibility of image misregistration between steady state and wash-out images. We use MRI compliant pneumotachometers to ensure similar inflation levels across breathholds. We have evaluated the impact of significant misregistration as show below for one of the subjects with COPD. Figure 7a shows the SS, washout and difference image. Figure $7 b$ shows a difference image where the in-plane wash-out image has been scaled up by $10 \%$ (analogous to breath-hold differences). Similar effects are seen for image translation (1 pixel LR and SI) seen in Figure 7c. It seems clear that such misregistration is easily seen at the edges of the images (but not in the original difference image) and the collateral ventilation areas persist in the lung field such that the misregistration is not the source of the apparent $\mathrm{CV}$ defects. We consider that validation of interlobar collateral ventilation could be approached in a study of subjects scheduled for lung volume reduction using endo-bronchial valve placement where invasive evaluation of $\mathrm{CV}$ is commonly used during the procedure. While intra-lobar CV remains interesting, it may be challenging to distinguish between very slow filling/emptying compartments and intra-lobar (intra-acinar) collateral ventilation as this transition may simply reflect a continuum of lung function in diseased lungs. These observations support the use of such an imaging strategy for evaluation of interlobar collateral ventilation in the context of patient evaluation for endobronchial valve placement for lung volume reduction.

\section{Acknowledgments}

The imaging data used in this project was obtained from subjects scanned associated with NHLBI R01-HL111294. The parent study was supported by NIH/NHLBI R01-HL111294 (NCT 01640288).

\section{Funding}

None. 


\section{Conflicts of interest}

No potential conflict of interest was reported by the authors.

\section{References}

1. Martin HB. Respiratory bronchioles as the pathway for collateral ventilation. J Appl Physiol. 1966;21:1443-1447.

2. Woolcock AJ, Macklem PT. Mechanical factors influencing collatera ventilation in human, dog, and pig lungs. J Appl Physiol. 1971;30:99115 .

3. Terry PB, Traystman RJ, Newball HH, et al. Collateral ventilation in man. N Engl J Med. 1978;298(1):10-15.

4. Inners CR, Terry PB, Traystman RJ, et al. Effects of lung volume on collateral and airways resistance in man. J Appl Physiol Respir Environ Exerc Physiol. 1979;46(1):67-73.

5. Cetti EJ, Moore AJ, Geddes D.M. Collateral ventilation. Thorax. 2006;61:371-373.

6. Higuchi T, Reed A, Oto T, et al. Relation of interlobar collaterals to radiological heterogeneity in severe emphysema. Thorax. 2006;61:409 413.

7. Lindskog GE. Collateral respiration in the normal and diseased lung. Yale J Biol Med. 1951;23:311-316.

8. Khauli S, Bolukbas S, Reed RM, et al. Interlobar collateral ventilation in severe emphysema. Thorax. 2016;71:1168-1169.

9. Bezzi M, Luzzi V, Novali M, et al. Competence in bronchoscopic treatments in emphysema. Panminerva Med. 2019;61(3):401-421.

10. de Oliveira HG, de Oliveira SM, Rambo RR, et al. Fissure integrity and volume reduction in emphysema: a retrospective study. Respiration. 2016;91(6):471-479.

11. Fiorelli A, Poggi C, Anile M, et al. Visual analysis versus quantitative CT analysis of interlobar fissure integrity in selecting emphysematous patients for endobronchial valve treatment. Interact Cardiovasc Thorac Surg. 2019;28(5):751-759.

12. Fiorelli A, Santini M, Shah P. When can computed tomography-fissure analysis replace Chartis collateral ventilation assessment in the prediction of patients with emphysema who might benefit from endobronchial valve therapy. Interact Cardiovasc Thorac Surg. 2018;26:313-318.

13. Gompelmann D, Eberhardt R, Slebos DJ, et al. Diagnostic performance comparison of the Chartis System and high-resolution computerized tomography fissure analysis for planning endoscopic lung volume reduction. Respirology. 2014;19:524-530.

14. Koster TD, Slebos DJ. The fissure: interlobar collateral ventilation and implications for endoscopic therapy in emphysema. Int J Chron Obstruct Pulmon Dis. 2016;11:765-773.

15. Robinson PD, Goldman MD, Gustafsson PM. Inert gas washout: theoretical background and clinical utility in respiratory disease. Respiration. 2009;78(3):339-355.

16. Darling RC, Cournand A, Richards DW. Studies on the intrapulmonary mixture of gases. III. An open circuit method for measuring residual air. J Clin Invest. 1940;19:609-618.
17. Ball WCJ, Stewart PB, Newshaml LG, et al. Regional pulmonary function studied with xenon 133. J Clin Invest. 1962;41:519-531.

18. West JB, Holland RA, Dollery CT, et al. Interpretation of radioactive gas clearance rates in the lung. J Appl Physiol. 1962;17:14-20.

19. Hoffman EA, Chon D. Computed tomography studies of lung ventilation and perfusion. Proc Am Thorac Soc. 2005;2:492-498.

20. van Beek EJ, Hoffman EA. Functional imaging: CT and MRI. Clin Chest Med. 2008;29:195-216.

21. Bauman G, Puderbach M, Deimling M, et al. Non-contrast-enhanced perfusion and ventilation assessment of the human lung by means of fourier decomposition in proton MRI. Magn Reson Med. 2009;62:656664

22. Chae EJ, Seo JB, Goo HW, et al. Xenon ventilation CT with a dualenergy technique of dual-source CT: initial experience. Radiology. 2008;248(2):615-624.

23. Conradi MS, Saam BT, Yablonskiy DA, et al. Hyperpolarized $3 \mathrm{He}$ and perfluorocarbon gas diffusion MRI of lungs. Progress in Nuclear Magnetic Resonance Spectroscopy. 2006;48:63-83.

24. Hachulla AL, Pontana F, Wemeau-Stervinou L, et al. Krypton ventilation imaging using dual-energy CT in chronic obstructive pulmonary disease patients: initial experience. Radiology. 2012;263:253-259.

25. Kauczor HU, Hanke A, Van Beek EJ. Assessment of lung ventilation by MR imaging: current status and future perspectives. Eur Radiol. 2002;12(8):1962-1970.

26. van Beek EJ, Wild JM, Kauczor HU, et al. Functional MRI of the lung using hyperpolarized 3-helium gas. J Magn Reson Imaging. 2004;20:540-554

27. Biederer J. in MRI of the Lung. 2017:1-20.

28. Marshall H, Collier GJ, Johns CS, et al. Imaging collateral ventilation in patients with advanced chronic obstructive pulmonary disease: relative sensitivity of $3 \mathrm{He}$ and $129 \mathrm{Xe}$ MRI. J Magn Reson Imaging. 2019;49(4):1195-1197.

29. Kirby M, Svenningsen S, Kanhere N, et al. Pulmonary ventilation visualized using hyperpolarized helium-3 and xenon-129 magnetic resonance imaging: differences in COPD and relationship to emphysema. J Appl Physiol. 2013;114(6):707-715.

30. Halaweish AF, Moon RE, Foster WM, et al. Perfluoropropane gas as a magnetic resonance lung imaging contrast agent in humans. Chest 2013;144(4):1300-1310

31. Ruiz-Cabello J, Barnett BP, Bottomley PA, et al. Fluorine (19F) MRS and MRI in biomedicine. NMR Biomed. 2011;24:114-129.

32. Jacob RE, Chang YV, Choong CK, et al. 19F MR imaging of ventilation and diffusion in excised lungs. Magn Reson Med. 2005;54(3):577-585.

33. Perez-Sanchez JM, Perez de Alejo R, Rodriguez I, et al. In vivo diffusion weighted 19F MRI using SF6. Magn Reson Med. 2005;54(2):460-463. 